\title{
Johannes Menke, Kontrapunkt II: Die Musik des Barock (= Grundlagen der Musik, Bd. 3), Laaber: Laaber 2017
}

Schlagworte/Keywords: Barock; Baroque; counterpoint; figured bass; Generalbass; Kontrapunkt; Lehrbuch; textbook

Die Reihe Grundlagen der Musik des LaaberVerlags steht ähnlich wie viele aktuelle Handbuch-Projekte vor der Aufgabe, mitunter äußerst heterogenes Wissen in verständlicher und kompakter Form nicht nur aufzubereiten, sondern auch mit einer narrativen Logik auszustatten, die nicht immer von selbst aus den geschilderten Gegenständen erwächst. Die einzelnen Bücher, die nach Auskunft des Verlags vor allem zur Begleitung des universitären Unterrichts gedacht sind, rekonstruieren demnach aus verschiedenen Momentaufnahmen des Forschungsstands der letzten 50 Jahre einen mehr oder weniger geschlossenen Status quo, auf dessen Grundlage erst die eigentliche Darstellung aufbauen kann. Da es sich um didaktische Literatur handelt, spiegeln die Bände aber auch Diskurse des Faches und verschiedene Versionen seiner Selbstdarstellung wider. Der innerfachliche Diskurs zum Thema sKontrapunkt und seine Vermittlung،, wie er sich spätestens seit Carl Dahlhaus' Untersuchungen über die Entstehung der harmonischen Tonalität ${ }^{1}$ entwickelt hat, lässt sich allerdings kaum homogenisieren. Gerade für ein so weites Thema wie die Musik des Barock ist eine Vermittlung zwischen Forschung und Lehre weder als geschlossenes Narrativ noch im Rahmen einer Systematik erschöpfend zu leisten.

In den letzten Jahren konnten sich eine ganze Reihe von Publikationen zur Musik des Barock als Standardwerke für den Unterricht etablieren, von denen viele ebenfalls didaktisch ausgerichtet und alle gut verständlich und zugänglich sind, darunter Robert O. Gjerdingens Music in the Galant Style, ${ }^{2}$ Thomas Daniels Der

\section{Dahlhaus 1968.}

2 Gjerdingen 2007. Menkes Buch endet zwar mit dem Übergang zum galanten Stil, dennoch überschneiden sich die satztechnischen Phänomene in weiten Bereichen.
Choralsatz bei Bach und seinen Zeitgenossen. Eine historische Satzlehre, ${ }^{3}$ dann Generalbasspraxis 1600-1800 ${ }^{4}$ von Siegbert Rampe innerhalb der Grundlagen-Reihe des Laaber-Verlags sowie die Arbeitsbücher zur Generalbasspraxis bei Giovanni Paisiello ${ }^{5}$ und Georg Friedrich Händel, ${ }^{6}$ die Johannes Menke mitherausgegeben hat. Parallel sind Lehrwerke zur Improvisation in historischen Stilen erschienen, ${ }^{7}$ historische Quellen wie Traktate von Joachim Burmeister, Wolfgang Caspar Printz, Johann David Heinichen, Friedrich Erhard Niedt, Friedrich Wilhelm Marpurg u. a. sind nicht nur problemlos oft auch online zugänglich, sondern von aktueller Sekundärliteratur ${ }^{8}$ begleitet, sodass im Unterricht ein informierter Umgang mit historischen Details möglich geworden ist, der noch um das Jahr 2000 herum nicht denkbar gewesen wäre.

Menke ist sich der Diversität der didaktischen Ansätze, aber auch der mitunter unvereinbaren Paradigmen kontrapunktischer Lehre ganz offenbar bewusst. Mit dem Untertitel Die Musik des Barock öffnet er nicht nur den Kontrapunkt-Begriff, der in den Curricula an Hochschulen für das Barockzeitalter bisher nahezu ausschließlich Fugenkontrapunkt umfasste, für andere Gattungen, sondern löst auch das Verständnis von Barock aus seiner in der deutschen und angelsächsischen Unterrichtspraxis

3 Daniel 2000

4 Rampe 2015

5 Holtmeier/Menke/Diergarten 2008.

6 Holtmeier/Menke/Diergarten 2013.

7 Bornstein 2001; Bornstein 2004; Schröder 2008; Erhardt 2011; Sanguinetti 2012; Moelants 2014 und andere Arbeiten aus dem Umfeld des Orpheus Institute und der Schola Cantorum Basiliensis.

8 Vgl. Scheideler/Wörner 2017; Ott/Roth/ Schlothfeldt (i. V.). 
gelegentlich zu engen Bindung an J.S. Bach und Händel: Der Schwerpunkt des Buches liegt eher im 17. als im 18. Jahrhundert, oder, wie Menke es im Vorwort ankündigt: „Das Ziel des vorliegenden Buches ist es, das Komponieren im Zeitraum zwischen 1600 und 1750 so breit wie möglich zu erfassen « (9).

Die inhaltliche Breite lässt kein stringentes Regelwerk zu - das Buch umfasst barocke Tonartenlehre, Generalbass-Topoi, Solmisation des 17. Jahrhunderts, Beispiele für konsonanten und dissonanten Satz, Kadenz-Typen als Werkzeuge musikalischer Gestaltung, Orgelpunkte, Formen der Syncopatio, Kanons und Sequenzen, die Oktavregel, chromatische Satztechniken, Verzierungspraxis, mehrfachen Kontrapunkt, contrapunti artificiosi (s. u.) und Cantusfirmus-Satz, das weite Gebiet der sogenannten Figurenlehre, Fuge, Komponieren mit OstinatoBässen, die (klavierpraktische) Erarbeitung von Tanzsätzen sowie die satztechnischen Standards der Triosonate und größerer Ensembles. So arbeitet Menke wie in der Vorgängerpublikation Kontrapunkt I: Die Musik der Renaissance ${ }^{9}$ ohne 'Verbotes und mit einem Minimum systematischer Regularien. Stattdessen wird versucht, den Leser*innen eine fast schon absurde Fülle von Material nahezubringen, was auf unterschiedliche Art und Weise geschieht.

Nach Menke ist das Buch "ein Lese- und Lehr-, weniger ein Arbeitsbuch « (10) - jedoch gestaltet sich das Lesen und Lernen je nach Gegenstand nicht immer gleich. Obwohl es sich um einen Textband ohne mediale Erweiterungen handelt, findet man sich beim Nachvollzug der dargestellten Phänomene bald mit abstrakten Notentexten (mehrfacher Kontrapunkt), bald mit dem Noten- und Partiturstudium (passim), bald am Klavier (Improvisation von Tanzsätzen und Generalbass-Fugen) beschäftigt. Äußerst gewinnbringend ist das Lesen in der Gruppe, also der Einsatz im Unterricht. Trotzdem stellt sich natürlich die Frage, was dieses Buch neben der handlichen Aufbereitung leistet, was die vom Umfang her weniger beengten Einzelpublikationen zum Generalbass, zur Figurenlehre, zum Partimento, zur Oktavregel, zur Fugenkomposition nicht leisten können: Es ist dies Menkes außerordentliche Qualität, die satztechnischen Phänomene mit- einander ins Spiel zu bringen. So werden über die interaktive Logik kontrapunktischer Strukturen satztechnische Topoi oder Pattern dort miteinander vernetzt, wo sie in Darstellungen wie Gjerdingens ${ }^{10}$ eher geschildert und in der Literatur nachgewiesen werden. Besonders gelungen ist die Vernetzung bei Menkes Ausführungen zu den verschiedenen Typen der cadenza doppia (58-62) sowie in dem Kapitel über Sequenzen (112-135). Ebenfalls innovativ ist die kontrapunktische Herangehensweise an die Oktavregel: Zeichnet sich in der etablierten Auffassung der regola dell'ottava entweder ein harmonisches Deutungsmuster $a b$, nach dem das Satzmodell als Linearisierung der Kadenz verstanden wird, oder eine generalbasspraktische Interpretation, die in den einzelnen 'Sitzen ‘ der Akkorde eine Alternative zum Akkorddenken des 19. Jahrhunderts sieht, so steuert Menke anhand der Darstellung der regola bei Antonio Filippo Bruschi einen Ansatz bei, der zwischen melodischen Standardformeln, ihrer Sequenzierung und ihrer algorithmischen Vernetzung mit dem Gang der Tonleiter vermittelt (99-105). Eine ähnlich sinnvolle Auflösung von Generalbass-Standards findet sich in der Diskussion der kontrapunktischen Herleitung des gängigen Septnonvorhaltsmodells ${ }^{11}$ bei der aufsteigenden Tonleiter, hier unter dem Aspekt steigender Sequenzen auf der Grundlage von Sekundkanons (129). In beiden Fällen vermittelt ein hörend-haptischer Zugang zwischen vertikalen und horizontalen Vorstellungen und bewirkt gleichsam ein Re-Inszenieren historischer Situationen, das eine wichtige Komponente in der Methodik des Buches bildet.

So lässt sich auch ein ganz anderes didaktisches Ethos als z. B. in Thomas Daniels Publikationen ausmachen: Während Daniel kategorisch die nicht als Originale belegten vierstimmigen Choralsätze der Bach-Schule aus dem Corpus ausschließt ${ }^{12}$ und dem Primat der Werktreue folgend kaum mehr als eine einzige schematische Sequenz als satztechnische Umsetzung beisteuert, ${ }^{13}$ mischt Menke die spielerisch-systematische Erklärung satztechnischer

10 Gjerdingen 2007.

11 So z. B. in Bach 1753, Teil 2, 328.

12 Daniel 2000, 10.

13 Ebd., 204. 
Gegebenheiten und Probleme mit historischen Kommentaren und Beispielen aus der Literatur, die mitunter über mehr als 100 Jahre gestreut sein können und so auf engem Raum etwa Claudio Monteverdi, Giacomo Carissimi, Johann Caspar Kerll, Henry Purcell und Johann Sebastian Bach verbinden (139-149). Man glaubt, 'Musik im Fluss` zu erleben, auch wenn die gewählten stilistischen Idiome eben nicht mit der Genauigkeit einer Phraseologie aufgelistet und festgehalten werden. Didaktisch und künstlerisch inspirierte Ideen wie z. B. die ohne Schlüssel und Akzidentien notierten Varianten mehrstimmig aufgefüllter Syncopationes, die am Klavier in konkrete Klänge gesetzt werden können (91-93), zeigen, dass es hier eben auch um eine Berührung mit historischem Material geht, das in der Umsetzung zwar flüchtig und kontrafaktisch, im Sinne experimenteller Archäologie aber nicht weniger historisch ist als der archivarische, dem Medium Schrift scheinbar (!) adäquatere Zugang.

\section{CLOSE-READING HISTORISCHER QUELLEN ALS METHODE}

Das Buch bildet an fünf Stellen Lektüre-Inseln aus: Passagen aus Antonio Berardis Documenti harmonici von 1687 (44ff. und 170-179), aus Christoph Bernhards Tractatus compositionis augmentatus aus der Mitte des 17. Jahrhunderts (192-196), aus Johann David Heinichens Kompositionslehre Der General-Bass in der Composition von 1728 (209-216) und schließlich aus der zweiten Auflage des zweiten Teils von Friedrich Ehrhardt Niedts Musicalischer Handleitung von 1721 (270-282) werden minutiös erörtert, auf satztechnische oder auch stilistische Probleme angewandt und für den Unterrichtsgebrauch aufbereitet.

Besonders hervorzuheben sind dabei - da die Technik in den aktuellen Curricula nicht sehr verbreitet ist - die Darlegungen bei Berardi zu den contrapunti artificiosi (170-179). Als Spur noch in der von Johann Joseph Fux geprägten Gattungslehre erhalten, bezeichnen contrapunti artificiosi eine Reihe etüdenhafter Auflagen, wie ein Kontrapunkt zu einem gegebenen Cantus firmus konsequent zu gestalten sei. Menke spricht treffend von einem "Design « (186), das sich in algorithmischer Wiederkehr (sogenannte perfidie oder obblighi ${ }^{14}$ ) von Pattern, darunter Syncopationes, horizontale und vertikale Intervallvorgaben, Intervall-Aussparungen, rhythmische Muster und ganze Phrasen, mit äußerster Konsequenz wiederholt. Das Kapitel demonstriert überzeugend und überaus relevant für die Unterrichtspraxis den Unterschied zwischen einer stilfreien Systematik, zu der sich die Fux'sche Gattungslehre leider entwickeln konnte, und einem stilgebundenen Idiom, dessen Ästhetik oder auch ars inventoria im Spannungsfeld zwischen Expressivität und Kombinatorik zu suchen ist.

Eine ganz andere Schulung erfahren die Leser*innen bei der kommentierten Lektüre von Niedts Musicalischer Handleitung (270-282). Trotz der Relevanz und Bekanntheit dieses historischen Lehrwerks ist Niedt für den Unterricht keine unproblematische Quelle: Nicht alle Probestücke sind musikalisch von akzeptabler Qualität, einige Gerüstsätze sind in ihrer harmonischen und linearen Logik so sperrig, dass jedwedes Nachschaffen oder Umsetzen der Aufgaben für die Studierenden frustrierend sein kann, und auch die penibel aufgelisteten Diminutionen und Figurationen sind nicht alle geeignet. Anders als bei Heinichen schützt hier die Zeitgenossenschaft nicht vor musikalischen Mängeln. Menkes Auswahl des schlichten, aber stimmigen bezifferten Bass-Modells für verschiedene Suiten-Sätze (270) ist demnach gut durchdacht und auch die von Niedt ausgeführten Beispiele bieten sehr schöne Gelegenheiten für den Vergleich mit der eigenen klavierpraktischen Umsetzung in drei- und mehrstimmige figurierte Sätze.

\section{BAROCKE >ÜBERFÜLLE}

Zum vertiefenden Studium figurativen Reichtums verweist Menke auf Wolfgang Caspar Printz' ausführliche Darstellung im zweiten Teil des Phrynis Mitilenaeus (198). Folgt man dieser Empfehlung trifft man auf eine von Paragraph zu Paragraph weniger zu überschauende Menge teils systematisch abgeleiteter, teils aus konkreter Diminutions- oder Gesangspraxis übernommener Motive. Die immer wieder erfolgenden Versuche, die riesige VokabelSammlung nach Intervallgröße, Bewegungs-

14 Vgl. auch Bornstein 2001, 162-180. 
richtung, Länge, Notenwert und anderen Kategorien fasslich zu machen, erhöht noch die beängstigende Überforderung und auch gelegentliche musikalische Irrelevanz der enzyklopädischen Anlage. Allein Printz' fast erschöpfte Formulierung "Aus diesem allem folget unfehlbar/daß ein Viertel-Schlag nur durch die in Fusen und Semifusen bestehenden Figuren 2897. mahl variret werden kan « ${ }^{15}$ zeigt, dass die Neigung zum vollständigen, welterklärenden sAuserzählen in barocken Lehrwerken einerseits demselben Geiste entspringt wie die Werke selbst (198), dass sie aber - wie auch das nach 120 Paragraphen in den eigenen Details ertrinkende Kapitel über musikalischen Stil bei Mattheson ${ }^{16}$ - nicht nur für heutige didaktische Bedürfnisse ein Problem der Vermittlung darstellen.

Trotz Menkes versierter Reduktion und gezielter Auswahl aus rund 40 Quellen bilden sich diese ,Überwerfungen lichkeit des Materials oder in 'Falten`, wie es Menke nach Gilles Deleuze nennt (17-19), auch in diesem Lehrwerk ab, wenn auch in ganz geringem Maße. Die fast immer an der Originalquelle erfolgte Begriffsklärung entspricht nur selten der Allgemeinheit der Phänomene. So ist z. B. die Definition der Heterolepsis nach Christoph Bernhard im Zusammenhang der latenten Argumentation dort passend als Sprung in die Dissonanz einer anderen Stimme beschrieben (64). Dass dieses Phänomen in nur wenig späteren Quellen ${ }^{17}$ etwas weiter gefasst wird und dass auch in der Folge des Buches bei der Darstellung der "theatralischen Dissonanzauflösung " mehrfach Fälle von Heterolepsis angeführt werden, wird zwar erwähnt (210), würde sich aber klarer einprägen, wären die Beispiele nicht durch fast 150 Seiten getrennt. Ebenso kann ein Pattern aus fallenden Quarten und steigenden Terzen im Unterquart-/Oberquint- oder Prim-/(Unter-)Oktavkanon immer mit einer absteigenden Tonleiter kontrapunktiert werden, die auch chromatisiert werden kann. Dieses Phänomen kommt an verschiedenen Stellen ins Spiel $(118,131,142,143,149)$, ohne dass diese Passagen wirklich miteinan-

15 Printz 1696, 61; und das ist erst eine eher am Anfang der Liste stehende Zwischenbilanz.

16 Mattheson 1739, 68-93.

17 Z. B. Walther 1955, 274. der kommunizieren würden, da der übergeordnete Aspekt jeweils ein anderer ist. ${ }^{18}$ In derlei Fällen ist das Sachregister des Bandes sehr nützlich.

\section{KANON-KORREKTUR}

Die Musik Johann Sebastian Bachs bleibt auch in diesem Lehrwerk präsent. Einerseits gibt es in fast jedem Kapitel einen expliziten Verweis auf sein Werk, andererseits scheinen auch einige der abstrakten Notenbeispiele vor dem Hintergrund 'großer Vorbilder entstanden zu sein, wie z. B. Abbildung 7.22 g die Zwischenspiele der Fuge in h-Moll BWV 869 zu zitieren scheint (130). Dennoch verlagert sich mit der gelegentlichen Konzentration auf französische (aber eben weniger auf Jean-Philippe Rameau) und italienische Literatur des 17. Jahrhunderts der rekonstruierte geschichtliche Raum weg von dem, was in der Lehre bisher weitgehend unter barockem Kontrapunkt verstanden wurde, nämlich - grob gesagt - die Fugen des Wohltemperierten Klaviers und die zwei- und dreistimmigen Inventionen in der theoretischen Rezeption durch Kirnberger und Marpurg oder noch späterer Quellen. Mit der entschiedenen Integration bisher weniger der Disziplin `Kontrapunkt zugeordneter Praktiken der Generalbass-Tradition zeichnet Menke ein indirektes Bild, so etwas wie ein Negativ des etablierten Lehrkanons, in dessen Zentrum die zu korrigierende retrospektive Idee einer Entwicklung vom modalen Intervallsatz der Renaissance zum sharmonischen Kontrapunktı des 18. Jahrhunderts steht. ${ }^{19}$ Dass dies nicht nur wesentlich heterogener als häufig dargestellt, sondern möglicherweise sogar überhaupt nicht in dieser im 20. Jahrhundert rekonstruierten Klarheit stattgefunden hat, wird durch die Dokumente und den von einer anachronistischen Idee von Tonalität wegführenden Gedankengang dieses Buches belegt.

Dabei sollte erwähnt werden, dass auch die zitierte Sekundärliteratur und die verwendete Begrifflichkeit einen weiten Bereich der Dis-

18 Hier wäre der Verweis auf andere aktuelle Literatur zu algorithmischen Techniken wie Prey (2012) oder Ott (2014) sicherlich sinnvoll gewesen.

19 Besonders stilisiert bei La Motte (1981, 221ff.). 
kussion der letzten Jahre auszusparen scheinen. Besonders deutlich wird dies bei der Vermeidung des Begriffs >Dur-Moll-Parallelismus` und seiner Varianten, die bei Menke nach der Improvisationspraxis des 16. Jahrhunderts »3-5Satz« oder »3-8-Satz « heißen und in einer Weise ausdifferenziert sind, dass die gewohnte (und nicht nur schlechte) Vereinheitlichung des Satzmodells hier kaum stattfindet. Auch wird fast alles vermieden, was mit dem in der Lehre etablierten Terminus sphrygische Wendung, verbunden ist, und zwar wird der Begriff nicht lediglich durch historisch belegte Nomenklatur wie z. B. „Clausula mi« ersetzt, sondern die hier aufgeführten Alternativen »Cadentia minor « oder „Cadentia minima « nach Georg Muffat (53-57) bezeichnen durch ihren geschichtlichen und stilistischen Kontext sowie durch ihre Rezeptionsgeschichte etwas anderes. Man hätte hier vielleicht etwas deutlicher auf Sekundärliteratur der letzten 40 Jahre ver-

\section{Literatur}

Bach, Carl Philipp Emanuel (1753), Versuch über die wahre Art, das Clavier zu spielen, Berlin: Christian Friedrich Henning, Reprint Wiesbaden: Olms 1986.

Bornstein, Andrea (2001), Two-Part Didactic Music in Printed Italian Collections of the Renaissance and Baroque (1521-1744), University of Birmingham Research Archive, E-Theses Repository. http://etheses.bham. ac.uk/677 (15.10.2018)

Bornstein, Andrea (2004), Two-Part Italian Didactic Music. Printed Collections of the Renaissance and Baroque (1521-1744), Bologna: UT Orpheus Editioni.

Dahlhaus, Carl (1968), Untersuchungen über die Entstehung der harmonischen Tonalität, Kassel: Bärenreiter.

Daniel, Thomas (2000), Der Choralsatz bei Bach und seinen Zeitgenossen. Eine historische Satzlehre, Köln: Dohr.

20 Meier 1974, 83 und passim; Meier 1992, 87; Williams 1997; Ringhandt 2001; Jeßulat 2001; Eder 2004. weisen können, ${ }^{20}$ sei es auch nur um der $A b-$ grenzung willen.

Insgesamt hat Menke ein überaus anregendes und informatives Lehrwerk vorgelegt, das in seiner Kürze und Praxisnähe ein ebenso historisch differenziertes wie im besten Sinne handwerklich vermitteltes Paradigma musiktheoretischer Lehre lebendig und motivierend umsetzt. Der abschließenden Aufforderung »das alte Terrain aufs Neue wiederzuentdecken « - und das »mit Chancen, Risiken und auch einem Schuss Abenteuer« (324) - folgt man gern. So sehr es zu begrüßen ist, dass überhaupt die Möglichkeit besteht, ein Lehrwerk mit einer solchen Menge von instruktiven Notenbeispielen herauszugeben, reichen das Lektorat und die editorische Aufbereitung der Notenbeispiele leider nicht an das hohe inhaltliche Niveau heran.

Ariane Jeßulat

Eder, Petrus (2004), Die modernen Tonarten und die phrygische Kadenz, Tutzing: Schneider.

Erhardt, Martin (2011), Improvisation mit Ostinatobässen aus dem 16. bis 18. Jahrhundert, Magdeburg: Walhall.

Gjerdingen, Robert O. (2007), Music in the Galant Style, New York: Oxford University Press.

Holtmeier, Ludwig / Johannes Menke / Felix Diergarten (Hg.) (2008), Giovanni Paisiello. Regole per bene accompagnare il partimento o sia il basso fondamentale sopra il Cembalo, Wilhelmshaven: Noetzel.

Holtmeier, Ludwig / Johannes Menke / Felix Diergarten (Hg.) (2013), Solfeggi, Bassi e Fughe. Georg Friedrich Händels Übungen zur Satzlehre, Wilhelmshaven: Noetzel.

Jeßulat, Ariane (2001), Die Frage als musikalischer Topos. Studien zur Motivbildung in der Musik des 19. Jahrhunderts, Sinzig: Studio. 
La Motte, Diether de (1981), Kontrapunkt. Ein Lese- und Arbeitsbuch, Kassel: Bärenreiter.

Mattheson, Johann (1739), Der vollkommene Kapellmeister, Hamburg: Herold, FaksimileNachdruck, 5. Auflage, Kassel: Bärenreiter 1991.

Meier, Bernhard (1974), Die Tonarten der klassischen Vokalpolyphonie, Utrecht: Oosthoek, Scheltema \& Holkema.

Meier, Bernhard (1992), Alte Tonarten, Bärenreiter: Kassel.

Menke, Johannes (2015), Kontrapunkt I: Die Musik der Renaissance, Laaber: Laaber.

Ott, Immanuel (2014), Methoden der Kanonkomposition bei Josquin Des Prez und seinen Zeitgenossen, Hildesheim: Olms.

Ott, Immanuel / Markus Roth / Matthias Schlothfeldt (i. V.), Komponieren im Barock, Laaber: Laaber.

Prey, Stefan (2012), Algorithmen zur Satztechnik und ihre Anwendung auf die Analyse, Phil. Diss., Universität Osnabrück. https://reposi torium.ub.uni-osnabrueck.de/handle/urn: nbn:de:gbv:700-2012102410434 (15.10.2018)
Printz, Wolfgang Caspar (1696), Phrynis Mitilenaeus, Dresden: Miedt und Zimmermann.

Rampe, Siegbert (2015), Generalbasspraxis 1600-1800, Laaber: Laaber.

Ringhandt, Ute (2001), Sunt lacrimae rerum. Untersuchungen zur Darstellung des Weinens in der Musik, Sinzig: Studio.

Scheideler, Ullrich / Felix Wörner (Hg.) (2017), Musiktheorie von der Antike bis zur Gegenwart (= Lexikon Schriften über Musik, Bd. 1), Kassel: Bärenreiter / Stuttgart: Metzler.

Walther, Johann Gottfried (1955), Praecepta der musicalischen Composition [1708], hg. von Peter Benary, Leipzig: Breitkopf \& Härtel.

Williams, Peter (1997), The Chromatic Fourth, Oxford: Clarendon Press.

Jeßulat, Ariane (2018): Johannes Menke, Kontrapunkt II: Die Musik des Barock (= Grundlagen der Musik, Bd. 3), Laaber: Laaber 2017. ZGMTH 15/2, 209-214. https://doi.org/10.31751/993

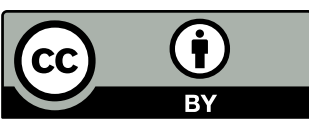

(C) 2018 Ariane Jeßulat (ajessulat@aol.com)

Dieser Text erscheint im Open Access und ist lizenziert unter einer Creative Commons Namensnennung 4.0 International Lizenz.

This is an open access article licensed under a

Creative Commons Attribution 4.0 International License.

eingereicht / submitted: 22/10/2018

angenommen / accepted: 23/10/2018

veröffentlicht / first published: 18/12/2018

zuletzt geändert / last updated: 18/12/2018 